\title{
Joint Iterative Detection and Decoding in the Presence of Phase Noise and Frequency Offset
}

\author{
Alan Barbieri, Student Member, IEEE, Giulio Colavolpe, and Giuseppe Caire, Fellow, IEEE
}

\begin{abstract}
We present a new algorithm for joint detection and decoding of iteratively decodable codes transmitted over channels affected by a time-varying phase noise (PN) and a constant frequency offset. The proposed algorithm is obtained as an application of the sum-product algorithm to the factor graph representing the joint a posteriori distribution of the information symbols and the channel parameters given the channel output. The resulting algorithm employs the soft-output information on the coded symbols provided by the decoder and performs forwardbackward recursions, taking into account the joint probability distribution of phase and frequency offset. We present simulation results for high-order coded modulation schemes based on low-density parity-check codes and serially concatenated convolutional codes, showing that, despite its low complexity, the algorithm is able to cope with a strong PN and a significant uncompensated frequency offset, thus avoiding the use of complicated data-aided frequency-estimation schemes operating on a known preamble. The robustness of the algorithm in the presence of a time-varying frequency offset is also discussed.
\end{abstract}

Index Terms-Detection and decoding in the presence of phase noise and frequency offset, factor graphs (FGs), iterative detection and decoding, low-density parity-check (LDPC) codes, serially concatenated convolutional codes (SCCCs), sum-product algorithm (SPA), turbo codes (TCs).

\section{INTRODUCTION}

$\mathbf{I}$ $\mathrm{N}$ RECENT burst-mode satellite communications based on powerful turbo codes (TCs), serially concatenated convolutional codes (SCCCs), or low-density parity-check (LDPC) codes, and thus working at a very low signal-to-noise ratio (SNR), efficient methods for phase and frequency estimation/compensation are necessary to cope with the instabilities of transmitter and receiver oscillators and a possible carrier-frequency Doppler shift. An example of such systems is represented by the second-generation satellite digital video broadcasting (DVB-S2) where, in addition, the use of LDPC-coded modulation schemes based on high-order constellations increases the sensitivity to phase and frequency instabilities.

Paper approved by C. Schlegel, the Editor for Coding Theory and Techniques of the IEEE Communications Society. Manuscript received April 15, 2005; revised December 31, 2005. This work was supported by the European Space Agency, ESA-ESTEC, Noordwijk, The Netherlands. This paper was presented in part at the IEEE International Conference on Communications, Seoul, Korea, May 2005.

A. Barbieri and G. Colavolpe are with the Department of Information Engineering, University of Parma, 43100 Parma, Italy (e-mail: barbieri@ tlc.unipr.it; giulio.colavolpe@unipr.it).

G. Caire is with the Viterbi School of Engineering, Electrical Engineering Department, University of Southern California, Los Angeles, CA 90089 USA (e-mail: caire@usc.edu).

Digital Object Identifier 10.1109/TCOMM.2006.887501
The problem of iterative detection and decoding in the presence of phase noise (PN) has been addressed by several authors in the last few years [1]-[14]. ${ }^{1}$ Among them, the scheme in [4]-[6] and [14], based on the expectation-maximization (EM) algorithm, can be adopted for both TCs and LDPC codes. More recently, through the use of factor graphs (FGs) and the application of the sum-product algorithm (SPA) [15], some new interesting algorithms have been proposed [7]-[13].2 In particular, in [13], following the approach in [7], a Bayesian algorithm for joint detection and decoding in the presence of strong PN has been described. This algorithm works on an FG representing both code constraints and channel statistics. The SPA is then used to implement the maximum a posteriori (MAP) symbol detection strategy. Since the channel parameters, which are continuous random variables (RVs), are explicitly represented in the graph, the application of the SPA becomes impractical. To solve this problem, the method of canonical distributions is adopted. In particular, a Tikhonov parameterization of the messages exchanged along the graph edges associated with continuous RVs representing the channel phase is adopted. The resulting algorithm is shown to perform as well as a finely discretized version of the continuous SPA, which can be considered as a benchmark for practically optimal performance, at extremely low complexity [13]. Thus, it represents an attractive solution when powerful turbo- or LDPC-coded modulations are transmitted in the presence of strong PN, as in the DVB-S2 system.

In the presence of a constant frequency offset, the performance of the above-mentioned algorithms rapidly degrades, especially for high-order modulations and long code-block lengths. To overcome this problem, a possible solution is the adoption of an explicit carrier-frequency estimator, based on a data-aided algorithm working on a known preamble [17], [18]. However, the desired estimation accuracy can be only achieved by using long preambles and an efficient, but computationally expensive, algorithm (e.g., the Rife and Boorstyn [19], the Luise and Reggiannini [20], or the Mengali and Morelli [21] algorithms), thus decreasing the effective spectral efficiency and increasing complexity. Moreover, the impact of strong PN on the quality of a conventional frequency estimator might be dramatic. Therefore, frequency offset, PN, and decoding must be handled jointly. The insertion of long preambles for frequency-estimation purposes can be avoided by resorting to the soft-decision-directed estimator described in [22], whose

\footnotetext{
${ }^{1}$ See [13] for a detailed literature survey.

${ }^{2}$ In [16], it has been shown that the EM algorithm can be formulated as a message-passing algorithm in FGs. It is, however, important to notice a major difference: the SPA propagates extrinsic a posteriori probabilities, while the EM algorithm propagates a posteriori probabilities.
} 
complexity, however, remains considerable. In addition, in this case, the algorithm accuracy is also affected by the presence of PN.

In this paper, we extend the Tikhonov algorithm in [13] such that, with a slight increase in complexity, it can cope with both a strong PN and a constant carrier-frequency offset. In fact, since the use of an algorithm for detection in the presence of $\mathrm{PN}$ is mandatory, we consider the possibility of avoiding the explicit frequency estimation by properly designing an extended detection algorithm, able to perform detection in the presence of a constant frequency offset also. We assume that the phase coherence over successive codewords cannot be maintained, so that the carrier phase and the frequency offset must be recovered on a codeword-by-codeword basis. The proposed algorithm makes use of the soft output provided by the iterative decoder, does not require the insertion of a preamble of known symbols, and, by nature, is robust to $\mathrm{PN}$.

The paper is structured as follows. In Section II, the adopted system model is described. The proposed detection algorithm is developed in Section III, and in the same section, some implementation aspects are discussed. In Section IV, the performance of the algorithm is assessed by means of computer simulations. In addition, a comparison with the performance that can be obtained by means of "classical" approaches, based on explicit frequency offset estimation and compensation, is also provided, and the robustness in the presence of a time-varying frequency offset (Doppler rate) is also considered. Finally, conclusions are drawn in Section V.

\section{SYSTEM MODEL}

We consider the transmission of a sequence of complex modulation symbols $\mathbf{c} \triangleq\left\{c_{k}\right\}_{k=0}^{K-1}$, belonging to an $M$-ary alphabet $\mathcal{X}$, over an additive white Gaussian noise (AWGN) channel affected by carrier PN and frequency offset. Symbols $c_{k}$ are linearly modulated. Assuming Nyquist transmitted pulses, matched filtering, and phase variations slow enough that no intersymbol interference arises, the discrete-time baseband received signal is given by

$$
r_{k}=c_{k} e^{j \theta_{k}}+w_{k}, \quad k=0,1, \ldots, K-1
$$

where $\boldsymbol{\theta} \triangleq\left\{\theta_{k}\right\}_{k=0}^{K-1}$ represent the samples of the time-varying channel phase, and the noise samples $\mathbf{w} \triangleq\left\{w_{k}\right\}_{k=0}^{K-1}$ are independent and identically distributed (i.i.d.), complex, circularly symmetric Gaussian RVs, each with mean zero and variance equal to $2 \sigma^{2}$. The discrete-time model (1) will be used for the algorithm derivation. In the simulation results, however, a more realistic continuous-time model will be adopted. We assume that the sequence $\mathbf{c}$ is a codeword of a channel code $\mathcal{C}$ constructed over the above-mentioned $M$-ary modulation constellation $\mathcal{X}$, and is obtained by encoding of a sequence of information symbols $\mathbf{a} \triangleq\left\{a_{k}\right\}$. We include possible pilot symbols (known to the receiver) and/or possible differential encoding as a part of the code $\mathcal{C}$. The encoding function mapping information sequences $\mathbf{a}$ into the codewords $\mathbf{c}$ will be denoted by $\eta_{\mathcal{C}}$.
In the derivation of the proposed algorithm, for the timevarying channel phase $\theta_{k}$ we assume a random-walk (Wiener) model plus a constant frequency offset

$$
\theta_{k+1}=\theta_{k}+\phi+\Delta_{k}
$$

where $\left\{\Delta_{k}\right\}$ are real i.i.d. Gaussian RVs with zero mean and standard deviation, known to the receiver, $\sigma_{\Delta},{ }^{3}$ and $\phi \triangleq 2 \pi \nu T$, where $\nu$ is the frequency offset and $T$ is the symbol interval. We assume that $\nu$ is constant over a codeword, uniformly distributed in $\left[-\nu_{0}, \nu_{0}\right]$ with $\nu_{0} T<1 / 2$, and changes independently from one codeword to another. Hence, $\phi$ is uniformly distributed within $\left[-\phi_{0}, \phi_{0}\right]$, where $\phi_{0}=2 \pi \nu_{0} T<\pi$. The frequency offset $\nu$ and the sequence of phase increments $\left\{\Delta_{k}\right\}$ are supposed independent, unknown to both transmitter and receiver, and statistically independent of $\mathbf{c}$ and $\mathbf{w}$.

\section{PROPOSED ALGORITHM}

In the following, we assume that the reader is familiar with the FG/SPA framework (described, for example, in the tutorial paper [15]). We also use the following notation: we denote by $g\left(\eta, \sigma^{2} ; x\right)$ a Gaussian distribution in $x$, with mean $\eta$ and variance $\sigma^{2}$, and by $t(\zeta ; x)$ a Tikhonov distribution in $x$ characterized by the complex parameter $\zeta$, i.e.,

$$
\begin{aligned}
g\left(\eta, \sigma^{2} ; x\right) & =\frac{1}{\sqrt{2 \pi \sigma^{2}}} e^{-\frac{(x-\eta)^{2}}{2 \sigma^{2}}} \\
t(\zeta ; x) & =\frac{1}{2 \pi \mathrm{I}_{0}(|\zeta|)} e^{\Re\left[\zeta e^{-j x}\right]}
\end{aligned}
$$

where $\mathrm{I}_{0}(x)$ is the zeroth-order modified Bessel function of the first kind. To implement the MAP symbol-detection strategy, it is necessary to compute the marginal a posteriori probabilities (APPs) $P\left(a_{k} \mid \mathbf{r}\right)$, where $\mathbf{r} \triangleq\left\{r_{k}\right\}_{k=0}^{K-1}$. This can be accomplished, in an efficient way, by applying the SPA to the FG representing the joint APP distribution function of $\mathbf{a}, \boldsymbol{\theta}$, and $\phi^{4}$

$$
\begin{aligned}
p(\mathbf{a}, \boldsymbol{\theta}, \phi \mid \mathbf{r}) \\
\quad \propto p(\mathbf{r} \mid \mathbf{a}, \boldsymbol{\theta}) p(\boldsymbol{\theta} \mid \phi) P(\mathbf{a}) p(\phi) \\
\quad=\chi\left[\mathbf{c}=\eta_{\mathcal{C}}(\mathbf{a})\right] p(\mathbf{r} \mid \mathbf{c}, \boldsymbol{\theta}) p(\boldsymbol{\theta} \mid \phi) P(\mathbf{a}) p(\phi) \\
\quad \propto \chi\left[\mathbf{c}=\eta_{\mathcal{C}}(\mathbf{a})\right] \prod_{k} p\left(r_{k} \mid c_{k}, \theta_{k}\right) p\left(\theta_{k} \mid \theta_{k-1}, \phi\right) p(\phi)
\end{aligned}
$$

where we have assumed that the information symbols are independent, uniformly, and identically distributed (hence, $P(\mathbf{a})=$ const. $)$, we have denoted by $\chi\left[\mathbf{c}=\eta_{\mathcal{C}}(\mathbf{a})\right]$ the code indicator function, equal to 1 if $\mathbf{c}$ is the codeword corresponding to a and to zero otherwise, and finally, due to the phase model (2), we have used the conditional Markov property $p\left(\theta_{k} \mid \theta_{k-1}, \ldots, \theta_{0}, \phi\right)=p\left(\theta_{k} \mid \theta_{k-1}, \phi\right)$ and discarded the term

${ }^{3}$ Note that since the channel phase is defined modulo $2 \pi$, the probability density function (pdf) $p\left(\theta_{k+1} \mid \theta_{k}, \phi\right)$ can be approximated as Gaussian only if $\sigma_{\Delta} \ll 2 \pi$.

${ }^{4}$ We use the proportionality relationship $f \propto g$, indicating that $f=a g$ for some real constant $a$, since the SPA is defined up to scaling its messages by positive factors, independent of the variables represented in the graph. 


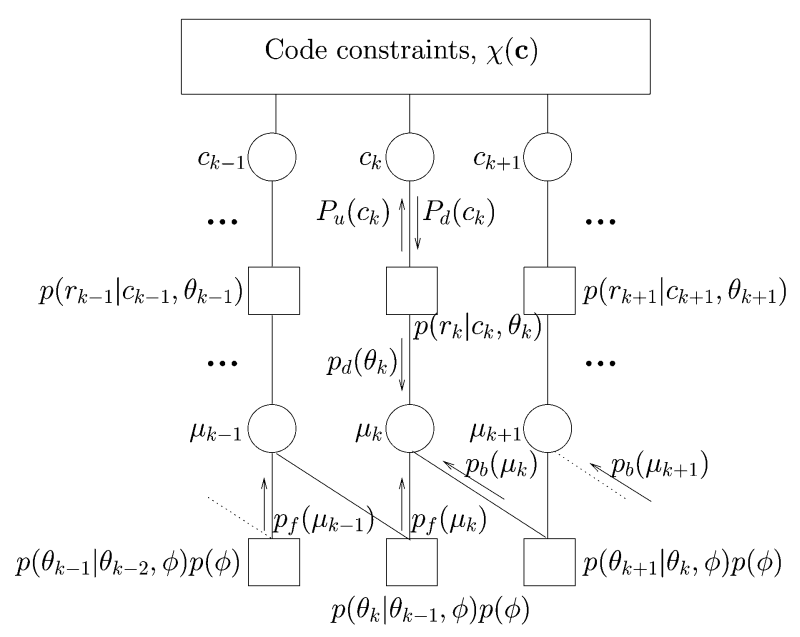

Fig. 1. FG after stretching.

$p\left(\theta_{0}\right)$ since $\theta_{0}$ is uniformly distributed. The pdf $p\left(\theta_{k+1} \mid \theta_{k}, \phi\right)$ can be further expressed as

$$
p\left(\theta_{k+1} \mid \theta_{k}, \phi\right)=g\left(\theta_{k}+\phi, \sigma_{\Delta}^{2} ; \theta_{k+1}\right)
$$

whereas the term $p\left(r_{k} \mid c_{k}, \theta_{k}\right)$ in (5) is given by

$$
\begin{aligned}
p\left(r_{k} \mid c_{k}, \theta_{k}\right) & \propto \exp \left\{-\frac{\left|r_{k}-c_{k} e^{j \theta_{k}}\right|^{2}}{2 \sigma^{2}}\right\} \\
& \propto e^{-\frac{\left|c_{k}\right|^{2}}{2 \sigma^{2}}} \exp \left\{\Re\left[\frac{r_{k} c_{k}^{*}}{\sigma^{2}} e^{-j \theta_{k}}\right]\right\} .
\end{aligned}
$$

The code constraint function $\chi(\mathbf{c})$ can be further factored in general [15]. The resulting function $p(\mathbf{a}, \boldsymbol{\theta}, \phi \mid \mathbf{r})$ in (5) can be represented by means of an FG. This FG representation of $p(\mathbf{a}, \boldsymbol{\theta}, \phi \mid \mathbf{r})$ has cycles of length four, even in the absence of cycles in the code representation. As a consequence, the application of the SPA to this FG leads to an algorithm which, in general, operates far from exact MAP decoding. With a slight increase in complexity, we may remove these cycles due to the channel model by stretching [15] the variable node $\phi$ to every variable node $\theta_{k}$, thus obtaining the equivalent FG represented in Fig. 1. The upper block is the graph representation of the adopted code and needs no further details here. In the figure, $\mu_{k}$ represents the "state" of the detector, i.e., $\mu_{k} \triangleq\left(\theta_{k}, \phi\right)$. In this new graph, we have now very long cycles due to the presence of the code and, as a consequence, there are good chances that the suboptimal iterative algorithm derived from the application of the SPA gives a good performance.

The application of the SPA leads to an iterative exchange of messages along the edges of the graph in Fig. 1. Some of these messages are represented in the figure. In particular, omitting for simplicity of notation the explicit reference to the current iteration, messages $P_{d}\left(c_{k}\right)$ and $P_{u}\left(c_{k}\right)$ are the APPs of coded symbols received from and sent to the decoder, respectively, whereas messages $p_{f}\left(\mu_{k}\right)$ and $p_{b}\left(\mu_{k}\right)$ represent a posteriori pdfs of $\mu_{k}$, given the past and the future respectively, i.e., ${ }^{5}$

$$
\begin{aligned}
& p_{f}\left(\mu_{k}\right) \triangleq p\left(\theta_{k}, \phi \mid \mathbf{r}_{0}^{k-1}\right)=p\left(\phi \mid \mathbf{r}_{0}^{k-1}\right) p\left(\theta_{k} \mid \phi, \mathbf{r}_{0}^{k-1}\right) \\
& p_{b}\left(\mu_{k}\right) \triangleq p\left(\theta_{k}, \phi \mid \mathbf{r}_{k+1}^{K-1}\right)=p\left(\phi \mid \mathbf{r}_{k+1}^{K-1}\right) p\left(\theta_{k} \mid \phi, \mathbf{r}_{k+1}^{K-1}\right)
\end{aligned}
$$

where the notation $\mathbf{r}_{i}^{j} \triangleq\left\{r_{k}\right\}_{k=i}^{j}, i<j$ is used. For the computation of these messages, we shall adopt a forward-backward schedule.

At this point, an implementation-complexity problem arises, due to the fact that messages $p_{f}\left(\mu_{k}\right)$ and $p_{b}\left(\mu_{k}\right)$ are continuous pdfs. In order to obtain practical algorithms, we adopt the canonical distribution approach described in [7]. First of all, we approximate $\phi$ as a discrete $\mathrm{RV}$ assuming $L$ values $\left\{\phi^{(\ell)}\right\}_{\ell_{\bar{K}}}^{L}$ in the interval $\left[-\phi_{0}, \phi_{0}\right]$. Hence, pdfs $p\left(\phi \mid \mathbf{r}_{0}^{k-1}\right)$ and $p\left(\phi \mid \mathbf{r}_{k+1}^{\bar{K}}-1\right)$ in (7) and (8) become probability mass functions (pmfs). These pmfs will be denoted by

$$
\begin{gathered}
P\left(\phi=\phi^{(\ell)} \mid \mathbf{r}_{0}^{k-1}\right) \triangleq \gamma_{f, k}^{(\ell)}, \quad \ell=1, \ldots, L \\
P\left(\phi=\phi^{(\ell)} \mid \mathbf{r}_{k+1}^{K-1}\right) \triangleq \gamma_{b, k}^{(\ell)}, \quad \ell=1, \ldots, L .
\end{gathered}
$$

In addition, the pdfs $p\left(\theta_{k} \mid \phi, \mathbf{r}_{0}^{k-1}\right)$ and $p\left(\theta_{k} \mid \phi, \mathbf{r}_{k+1}^{K-1}\right)$ are constrained to be in the family of Tikhonov distributions, i.e.,

$$
\begin{aligned}
p\left(\theta_{k} \mid \phi=\phi^{(\ell)}, \mathbf{r}_{0}^{k-1}\right) & \simeq t\left(a_{f, k}^{(\ell)} ; \theta_{k}\right) \\
p\left(\theta_{k} \mid \phi=\phi^{(\ell)}, \mathbf{r}_{k+1}^{K-1}\right) & \simeq t\left(a_{b, k}^{(\ell)} ; \theta_{k}\right)
\end{aligned}
$$

where $a_{f, k}^{(\ell)}$ and $a_{b, k}^{(\ell)}$ are proper parameters. The reason for this choice is explained in [13]. Hence, in order to update the messages by following the SPA, we have simply to forward parameters $\gamma_{f, k}^{(\ell)}, \gamma_{b, k}^{(\ell)}, a_{f, k}^{(\ell)}$, and $a_{b, k}^{(\ell)}$, instead of continuous pdfs.

Before trying to recursively update $p_{f}\left(\mu_{k}\right)$ and $p_{b}\left(\mu_{k}\right)$, we need to introduce a further approximation on the message $p_{d}\left(\theta_{k}\right)$ shown in Fig. 1. Based on the SPA rules, this message should be evaluated as

$$
p_{d}\left(\theta_{k}\right)=\sum_{c \in \mathcal{X}} p\left(r_{k} \mid c_{k}=c, \theta_{k}\right) P_{d}\left(c_{k}=c\right) .
$$

Based on the same min-divergence Gaussian approximation used in [13], we approximate this message as

$$
p_{d}\left(\theta_{k}\right) \simeq t\left(u_{k} ; \theta_{k}\right)
$$

where

$$
\begin{aligned}
& u_{k} \triangleq \frac{2 r_{k} \alpha_{k}^{*}}{2 \sigma^{2}+\beta_{k}-\left|\alpha_{k}\right|^{2}} \\
& \alpha_{k} \triangleq \sum_{c \in \mathcal{X}} c P_{d}\left(c_{k}=c\right) \\
& \beta_{k} \triangleq \sum_{c \in \mathcal{X}}|c|^{2} P_{d}\left(c_{k}=c\right) .
\end{aligned}
$$

${ }^{5}$ Obviously, this statement is exact in the absence of cycles in the overall graph, or approximate otherwise. 
By applying the SPA and the above-mentioned forward-backward schedule, we can now find the expressions to recursively update messages $p_{f}\left(\mu_{k}\right)$ and $p_{b}\left(\mu_{k}\right)$. Let us focus on the forward recursion. By considering that $\phi$ is a discrete variable, using (14), and discarding irrelevant multiplicative terms, we find

$$
\begin{aligned}
p_{f} & \left(\theta_{k+1}, \phi^{(\ell)}\right) \\
& =\int_{-\pi}^{\pi} p_{f}\left(\theta_{k}, \phi^{(\ell)}\right) p_{d}\left(\theta_{k}\right) p\left(\theta_{k+1} \mid \theta_{k}, \phi^{(\ell)}\right) d \theta_{k} \\
& =\int_{-\pi}^{\pi} p_{f}\left(\theta_{k}, \phi^{(\ell)}\right) t\left(u_{k} ; \theta_{k}\right) p\left(\theta_{k+1} \mid \theta_{k}, \phi^{(\ell)}\right) d \theta_{k} .
\end{aligned}
$$

All the functions involved in the integration are $2 \pi$-periodic in $\theta_{k}$, and the integrals may be extended over any interval of length $2 \pi$. Substituting (6), (7), (9), and (11) into (18), we obtain

$$
\begin{aligned}
\gamma_{f, k+1}^{(\ell)} t\left(a_{f, k+1}^{(\ell)} ; \theta_{k+1}\right) \simeq \gamma_{f, k}^{(\ell)} & \int t\left(a_{f, k}^{(\ell)} ; \theta_{k}\right) t\left(u_{k} ; \theta_{k}\right) \\
& \times g\left(\theta_{k}+\phi^{(\ell)}, \sigma_{\Delta}^{2} ; \theta_{k+1}\right) d \theta_{k} .
\end{aligned}
$$

By considering the facts in the Appendix, we finally find

$$
\begin{aligned}
\gamma_{f, k+1}^{(\ell)} t\left(a_{f, k+1}^{(\ell)} ; \theta_{k+1}\right) & \propto \gamma_{f, k}^{(\ell)} \frac{\mathrm{I}_{0}\left(\left|a_{f, k}^{(\ell)}+u_{k}\right|\right)}{\mathrm{I}_{0}\left(\left|a_{f, k}^{(\ell)}\right|\right) \mathrm{I}_{0}\left(\left|u_{k}\right|\right)} \\
& \times t\left(\frac{\left(a_{f, k}^{(\ell)}+u_{k}\right) e^{j \phi^{(\ell)}}}{1+\sigma_{\Delta}^{2}\left|a_{f, k}^{(\ell)}+u_{k}\right|} ; \theta_{k+1}\right) .
\end{aligned}
$$

We now need to choose $\gamma_{f, k+1}^{(\ell)}$ and $a_{f, k+1}^{(\ell)}$ for which (19) is satisfied (up to an irrelevant multiplicative factor). To simplify the computation, we can use the approximation for large arguments of the modified Bessel function, i.e., $\mathrm{I}_{0}(x) \simeq e^{x} / \sqrt{2 \pi x}$ and further neglect $\sqrt{2 \pi x}$ with respect to $e^{x}$. With this approximation, it is easy to find the coefficient updating recursive rule

$$
\begin{aligned}
a_{f, k+1}^{(\ell)} & =\frac{a_{f, k}^{(\ell)}+u_{k}}{1+\sigma_{\Delta}^{2}\left|a_{f, k}^{(\ell)}+u_{k}\right|} e^{j \phi^{(\ell)}} \\
\gamma_{f, k+1}^{(\ell)} & =\gamma_{f, k}^{(\ell)} \exp \left\{\left|a_{f, k}^{(\ell)}+u_{k}\right|-\left|a_{f, k}^{(\ell)}\right|\right\}
\end{aligned}
$$

where the term $\exp \left\{-\left|u_{k}\right|\right\}$ has been discarded, since independent of the variables represented in the graph. The previous equations give the forward recursions for coefficients $\gamma_{f, k}^{(\ell)}$ and $a_{f, k}^{(\ell)}, \ell=1, \ldots, L$. Similarly, for the backward recursion, we have

$$
\begin{aligned}
& a_{b, k-1}^{(\ell)}=\frac{a_{b, k}^{(\ell)}+u_{k}}{1+\sigma_{\Delta}^{2}\left|a_{b, k}^{(\ell)}+u_{k}\right|} e^{-j \phi^{(\ell)}} \\
& \gamma_{b, k-1}^{(\ell)}=\gamma_{b, k}^{(\ell)} \exp \left\{\left|a_{b, k}^{(\ell)}+u_{k}\right|-\left|a_{b, k}^{(\ell)}\right|\right\} .
\end{aligned}
$$

Both recursions require proper initialization. In particular, we have

$$
\begin{array}{cc}
a_{f, 0}^{(\ell)}=0 & a_{b, K-1}^{(\ell)}=0 \\
\gamma_{f, 0}^{(\ell)}=\text { const. } & \gamma_{b, K-1}^{(\ell)}=\text { const. }
\end{array} \quad \forall \ell=1, \ldots, L
$$

where "const." is any positive constant.

It is worth noting the roles of the evaluated coefficients: the complex parameter $a_{f, k}^{(\ell)}$ is related to an estimate of the phase $\theta_{k}$, given the past samples $\mathbf{r}_{0}^{k-1}$ and the frequency offset $\phi=\phi^{(\ell)}$. In particular, the argument of $a_{f, k}^{(\ell)}$ represents the mean value of this estimate, while its magnitude is (approximately) inversely proportional to the variance. Parameter $\gamma_{f, k}^{(\ell)}$ is proportional to the probability that $\phi$ is equal to the $\ell$ th quantization level $\phi^{(\ell)}$, given the samples $\mathbf{r}_{0}^{k-1}$. Practically, it is like having $L$ parallel detectors, each of them trying to keep track of the time-varying phase, assuming a different known frequency offset. The same observations hold for the backward parameters $a_{b, k}^{(\ell)}$ and $\gamma_{b, k}^{(\ell)}$, with the difference that they operate conditionally on the future instead of on the past.

After updating all forward and backward parameters, it is possible to update the probabilities $P_{u}\left(c_{k}\right)$ for coded symbols, which will be passed to the decoder. ${ }^{6}$ By applying the SPA, we find

$$
\begin{aligned}
P_{u}\left(c_{k}\right)= & \sum_{\ell} \int p_{f}\left(\theta_{k}, \phi^{(\ell)}\right) p_{b}\left(\theta_{k}, \phi^{(\ell)}\right) p\left(r_{k} \mid c_{k}, \theta_{k}\right) d \theta_{k} \\
\propto & e^{-\frac{\left|c_{k}\right|^{2}}{2 \sigma^{2}}} \sum_{\ell} \gamma_{f, k}^{(\ell)} \gamma_{b, k}^{(\ell)} \\
& \times \int t\left(a_{f, k}^{(\ell)} ; \theta_{k}\right) t\left(a_{b, k}^{(\ell)} ; \theta_{k}\right) e^{\Re\left[\frac{r_{k} c_{k}^{*}}{\sigma^{2}} e^{-j \theta_{k}}\right]} d \theta_{k}
\end{aligned}
$$

and by simple manipulations, we have

$$
P_{u}\left(c_{k}\right) \propto e^{-\frac{\left|c_{k}\right|^{2}}{2 \sigma^{2}}} \sum_{\ell} \gamma_{f, k}^{(\ell)} \gamma_{b, k}^{(\ell)} \frac{\mathrm{I}_{0}\left(\left|a_{f, k}^{(\ell)}+a_{b, k}^{(\ell)}+\frac{r_{k} c_{k}^{*}}{\sigma^{2}}\right|\right)}{\mathrm{I}_{0}\left(\left|a_{f, k}^{(\ell)}\right|\right) \mathrm{I}_{0}\left(\left|a_{b, k}^{(\ell)}\right|\right)} .
$$

To simplify the algorithm, in this case, we can also use the approximation $\mathrm{I}_{0}(x) \simeq e^{x}$.

To summarize, one iteration of the proposed joint detector/ decoder is formed by the following basic steps:

Step 1) evaluate coefficients $u_{k}, k=0,1, \ldots, K-1$, using the a priori information about code symbols (15), (16), and (17);

Step 2) recursively update forward and backward parameters $a_{f, k}^{(\ell)}, \gamma_{f, k}^{(\ell)}, a_{b, k}^{(\ell)}$, and $\gamma_{b, k}^{(\ell)}(20)$ and (21);

Step 3) evaluate a posteriori output probabilities $P_{u}\left(c_{k}\right)$ (22);

Step 4) update $P_{d}\left(c_{k}\right)$ from $P_{u}\left(c_{k}\right)$ applying (possibly several iterations of) the soft-in soft-out decoder for code $\mathcal{C}$.

${ }^{6}$ This decoder will be the specific soft-input soft-output decoder for the considered code. 
The computational complexity of the algorithm is linear in both parameters $K$ and $L$. More precisely, let us consider as a comparison, the standard Tikhonov algorithm [13], which is a special case (for $L=1$ ) of the algorithm described in this paper. With respect to the standard Tikhonov algorithm, there are now $L$ forward and backward parameters to be computed and, in addition, the evaluation of the a posteriori output probabilities $P_{u}\left(c_{k}\right)$ requires a sum of $L$ contributions. Step 1 remains unchanged. When $M \gg L$, we may assume, in practice, that the complexity is the same as that of the standard Tikhonov algorithm, since step 1 is computationally more intensive. However, when $M \ll L$, we may assume that the complexity is, roughly speaking, $L$ times that of the standard Tikhonov algorithm.

We conclude with some observations and remarks regarding the proposed algorithm.

Schedule. When a large value of $K$ is used, a pilot field is, in general, periodically inserted in the codeword, as in the DVB-S2 standard. Although the algorithm has been described by considering a forward-backward schedule, performed over the overall codeword, in this case, a mixed serial/parallel (S/P) schedule can be adopted, performing independent and parallel forward-backward recursions between pilot fields. In other words, the codeword is divided into subblocks with a pilot field at their start and end, and independent instances of the algorithm are applied in parallel to them. The resulting performance will be slightly degraded with respect to the above-mentioned forward-backward schedule, especially when these subblocks are very short, but the degree of parallelism of the implementation can be increased.

Role of $\sigma_{\Delta}$. The algorithm is obtained by assuming a Wiener model for the PN. In some applications, as, for example, in the DVB-S2 system [13], [23], this model does not hold. However, the derived algorithm still works well, as verified in the simulation results. In this case, the value of $\sigma_{\Delta}$ has to be optimized by computer simulations in order to maximize the performance.

Choice of the values $\phi^{(\ell)}$. In the derivation of the algorithm, no constraints have been given regarding the position of the quantization levels $\phi^{(\ell)}$. They do not need to be equally spaced, but may have arbitrary positions, also different from one iteration to the others. When the only a priori information regarding the frequency offset is that $\phi$ is uniformly distributed in $\left[-\phi_{0}, \phi_{0}\right]$, then the most obvious choice seems, at least at the first iteration, to use $L$ equally spaced quantization levels. From the second iteration onward, we verified by computer simulations that it is sufficient to keep only three adjacent quantization levels. In particular, defining

$$
\gamma^{(\ell)}=\gamma_{f, K-1}^{(\ell)}+\gamma_{b, 0}^{(\ell)}
$$

and considering at the end of the first iteration

$$
\hat{\ell}=\arg \max _{\ell} \gamma^{(\ell)}
$$

we keep the quantization levels corresponding to $\hat{\ell}-1, \hat{\ell}$, and $\hat{\ell}+1$. These three quantization levels are not kept constant for the successive iterations, but rigidly increased or decreased by the same quantity $\epsilon>0$, if $\gamma^{(\hat{\ell}+1)}>\gamma^{(\hat{\ell}-1)}$ or $\gamma^{(\hat{\ell}+1)}<\gamma^{(\hat{\ell}-1)}$, respectively. So the relative distance between these quantization levels is kept constant. The value of $\epsilon$, which is a fraction of the initial quantization step, is decreased with the iterations. Other strategies can be conceived in order to choose the position of the three quantization levels used from the second iteration onward; for example, inspired by the bisection method. In all cases, since a large number of quantization levels is used in the first iteration only, the complexity of the algorithm is practically equivalent to the case where we have only $L=3$ levels.

\section{Simulation Results AND Discussion}

In this section, the performance of the proposed algorithm is assessed by computer simulations in terms of bit-error rate (BER) versus $E_{b} / N_{0}, E_{b}$ being the received signal energy per information bit and $N_{0}$ the one-sided noise power spectral density. We assume the transmitter and receiver filters have squareroot raised-cosine frequency response with rolloff 0.5 . However, for the considered values of PN and uncompensated frequency offset, no difference has been observed on the receiver performance using different values of the filter rolloffs.

In order to assess the performance of the algorithm for different codes, modulation formats, and operating conditions, we consider the following coded modulation schemes. Code $\mathcal{C}_{1}$ is a rate-1/2 (3,6)-regular LDPC code, with codewords of length 4000 [24] and mapped onto a quadrature phase-shift keying (QPSK) modulation. In each codeword, 1 pilot every 20 code symbols has been inserted. A maximum of 40 iterations of the SPA on the overall graph is allowed. Codes $\mathcal{C}_{2}$ and $\mathcal{C}_{3}$ are two irregular LDPC codes with codewords of length 64800 specified in the DVB-S2 standard [25]. Code $\mathcal{C}_{2}$ has rate $4 / 5$ and is mapped onto an amplitude phase-shift keying (APSK) modulation with $M=32$ (32-APSK) [25, p. 27]. In this case, 1 pilot every 40 code symbols has been inserted, and a maximum of 40 iterations of the SPA on the overall graph is allowed. This latter simulation setting is very similar to that of the DVB-S2 standard, except for the pilot distribution, which was originally designed to be of 36 pilots every 1440 code symbols (same overhead). In our simulations, the pilot distribution has been changed because the original specification is not well suited for frequency compensation [22]. Code $\mathcal{C}_{3}$ has rate $2 / 3$, is mapped onto a PSK modulation with $M=8$ (8-PSK), and 1 pilot every 20 code symbols has been inserted. Since we consider LDPC codes, the decoder deriving from the application of the SPA to the Tanner graph of the code, i.e., the belief propagation decoder [15], is employed at the receiver.

In all cases, pilot symbols are inserted in the transmitted codewords in order to make the iterative detection algorithm bootstrap [13]. The insertion of pilot symbols corresponds to a decrease in the effective transmission rate, resulting in an increase in the required SNR which has been also artificially introduced in the curves labeled "known phase" (which correspond to the performance of an ideal coherent receiver which perfectly knows the PN and the frequency offset for the same code and modulation format) for the sake of comparison. In other words, the gap between the "known phase" curves and the others is uniquely due to the need for phase/frequency estimation/compensation, and not to the rate decrease due to pilot symbols which has been also considered for the "known phase" curves. In all simulation results, the time-varying PN is modeled according to the DVB-S2 compliant model for a baud 


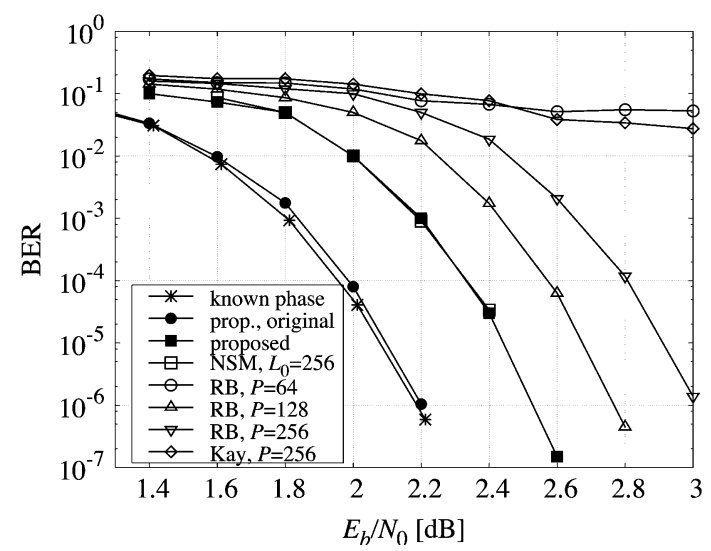

Fig. 2. Performance comparison for code $\mathcal{C}_{1}$. The original forward-backward schedule and the mixed S/P schedule are considered.

rate of $10 \mathrm{MBd}$, described in [13] and [23]. Parameter $\sigma_{\Delta}$ has been chosen equal to 0.3 degrees.

In Fig. 2, code $\mathcal{C}_{1}$ is considered. For the proposed algorithm, we show the performance in the case of the original forward-backward schedule (curve with label "original") and when the above-mentioned mixed S/P schedule is adopted on independent subblocks of length 42 symbols. We consider the presence of an uncompensated frequency offset uniformly distributed in the interval $\left[-2 \cdot 10^{-2}, 2 \cdot 10^{-2}\right]$. This range practically corresponds to the maximum range of resolvable frequency by using a data-aided algorithm working on the considered pilot distribution of 1 pilot every 20 code symbols. In fact, when the pilots are uniformly spaced with period $N=20$ symbols, then any normalized frequency offset larger than $1 / 2 N=2.5 \cdot 10^{-2}$ will be aliased [26]. The proposed algorithm works with 11 quantization levels in the first iteration, reduced to 3 from the second iteration onward. We also computed the performance of the Tikhonov algorithm, using both mentioned schedules, in the hypothesis of perfect knowledge of the frequency offset, obtaining two performance curves identical to that of the proposed algorithm, and for this reason, not shown in this figure. Hence, we can conclude that the proposed algorithm is able to effectively compensate the effect of a constant frequency offset, and its performance is as good as the practically best-known performance of joint detection and decoding in PN only (see extensive comparisons in [13]). In addition, we can say that the mixed S/P schedule produces a performance loss of about $0.3 \mathrm{~dB}$ that can be reduced, considering longer subblocks.

Let us now discuss the "classical" alternatives to the proposed receiver. We assume that the Tikhonov algorithm with mixed $\mathrm{S} / \mathrm{P}$ schedule (with subblocks of length 42 symbols) is used to cope with the PN, since in [13], it is shown that this algorithm can be regarded as the benchmark as far as PN alone is concerned. In order to estimate and to compensate the frequency offset, a data-aided frequency estimator, working on the pilot symbols, cannot be used since, due to PN, the phase on pilots uniformly spaced with period $N$ symbols tends to become uncorrelated when $N$ increases, thus affecting the estimation accuracy. So we append to each codeword a preamble of $P$ symbols for frequency-estimation purposes. For a given frequency-estimation algorithm, the value of $P$ must be optimized. In fact, due to PN, the frequency-estimation accuracy does not increase indefinitely with $P$, whereas the preamble insertion loss increases with $P$. Regardless of the value of $P$ (in the figure, the value of $P=256$ is shown), a simple estimator, such as the Kay algorithm [27], does not give a satisfactory performance, since at the operating SNR, it works below threshold [17], [18]. For the best (i.e., maximum-likelihood if $\mathrm{PN}$ was not present) estimator, i.e., the Rife and Boorstyn [19] (RB in the figure), for $P=64$ we are below threshold, whereas the optimal preamble length is around $P=128$. In this case, the loss with respect to the proposed receiver is only due to the preamble insertion loss.

Apart from the performance advantage and the absence of preamble, the proposed algorithm is also less complex. The increase in complexity of the proposed algorithm is distributed along the iterations. Hence, in a very-large-scale integration (VLSI) implementation, a little increased complexity burden is required per iteration, and the same hardware is used in the successive iterations. In addition, the latency with respect to the standard Tikhonov algorithm remains practically the same. On the contrary, the implementation of the RB algorithm will require a specific hardware to be used at the first iteration only, and the receiver latency will be increased.

We now consider the more sophisticated iterative soft-decision-directed frequency estimator recently proposed by Noels, Steendam, and Moeneclaey (NSM in the figure) [22] coupled with the Tikhonov algorithm working with the mixed S/P schedules on subblocks of 42 symbols. Instead of the soft decisions used in [22], we use the more reliable soft decisions produced by the decoder. Although the frequency estimate is constant, and thus, a single estimate could, in principle, be used, we apply different instances of the algorithm on subblocks of the transmitted codeword of length $L_{0}$ and derive a different frequency estimate for each block. At each iteration, these frequency estimates are updated using the refined soft decisions of the decoder. The reason for using these multiple estimates is twofold. First of all, we want to mitigate the effect of the PN on the frequency estimation accuracy. The other reason is that due to the very low operating SNR, the estimator can produce an outlier. In the presence of multiple estimates over a codeword, the decoder is still able to converge, despite that for one or a few subblocks we could have a wrong estimate, especially at the first iterations. The value of $L_{0}$ has been optimized by computer simulations and $L_{0}=256$ adopted.

Although this algorithm is much more complex with respect to the proposed one, it may be observed from Fig. 2 that there is no performance improvement. To understand this behavior, let us compare these two solutions from the point of view of the produced frequency estimates at the last iteration. The proposed algorithm does not produce an explicit frequency estimate. In fact, it (approximately) estimates the frequency a posteriori pdf. We assume as a frequency estimate the mean value of this pdf. In Fig. 3 we show, for the same simulation setting of the previous figure, the mean-square estimation error (MSEE), computed with reference to the symbol-rate normalized frequency, for the proposed receiver based on the original forward-backward schedule as well as for the mixed S/P schedule, and for the receiver based on the Tikhonov algorithm coupled with the NSM estimator. As intuitively expected, the proposed algorithm 


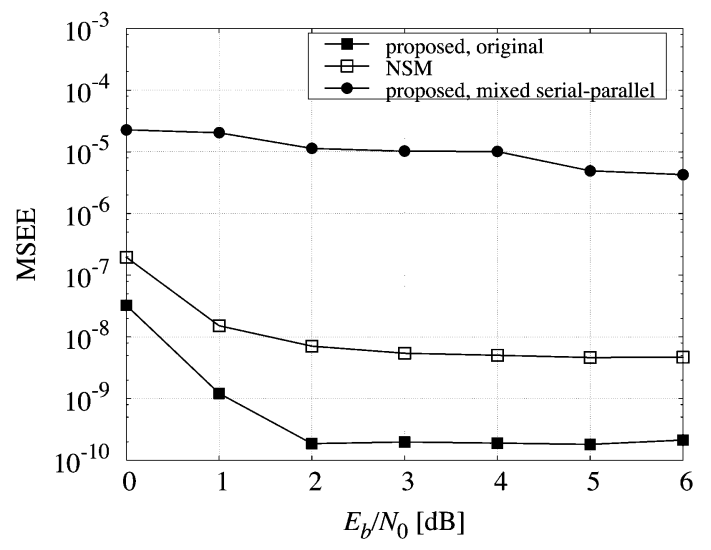

Fig. 3. MMSE for the NSM estimator and the proposed algorithm. The original forward-backward schedule and the mixed S/P schedule are considered.

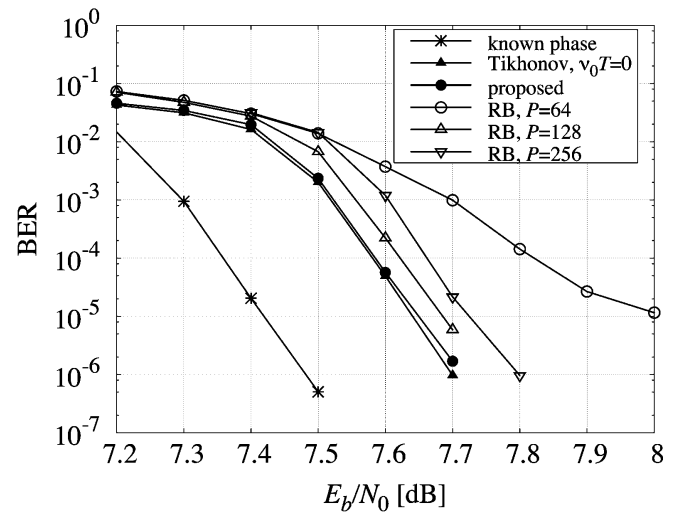

Fig. 4. Performance comparison for $\operatorname{code} \mathcal{C}_{2}$. The original forward-backward schedule is considered.

with the original forward-backward schedule outperforms the other solutions, since it computes the frequency estimate by observing the whole codeword and not only a subblock of length 256 symbols, as for the NSM algorithm, or of length- 42 symbols as for the proposed algorithm with mixed S/P schedule. However, in all cases, the frequency-estimation accuracy is sufficient to produce no performance loss in terms of BER, with respect to the corresponding performance of the Tikhonov algorithm with perfect knowledge of the frequency offset. The error floors that can be observed in the MSEE curves are due to the PN [28]. Hence, the proposed algorithm performs an implicit, iterative, low complexity, but very accurate frequency estimate.

These considerations extend to different codes and operating conditions. In Fig. 4, code $\mathcal{C}_{2}$ is considered and the frequency offset is uniformly distributed in the interval $\left[-10^{-2}, 10^{-2}\right]$. In this case also, the proposed receiver, based on 11 equally spaced quantization levels at the first iteration, and then, from the second iteration onward, on 3 variable quantization levels, is able to perfectly cope with the frequency offset, since there is no performance loss with respect to the Tikhonov algorithm working for $\nu_{0} T=0$. In this and the following figures, the optimal forward-backward schedule has been adopted. For the RB algorithm, operating on a known preamble of $P$ symbols, with the optimal value of $P=128$ there is still a performance loss with respect to the proposed algorithm.

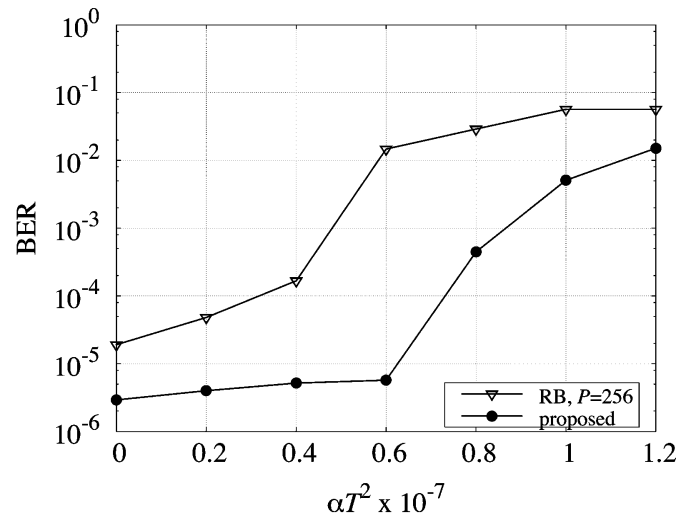

Fig. 5. Sensitivity to Doppler rate for code $\mathcal{C}_{3}$ and $E_{b} / N_{0}=3.9 \mathrm{~dB}$. The original forward-backward schedule is considered.

Since the proposed algorithm is able to effectively compensate a constant frequency offset, in Fig. 5, we finally consider the robustness of the proposed receiver in the presence of a time-varying frequency offset. This situation occurs, for instance, in satellite communication systems based on a low-earth-orbit (LEO) constellation, where the speed of each satellite relative to earth is rapidly time-varying. We use the commonly adopted assumption that the channel phase varies as $2 \pi\left[\nu T k+(1 / 2) \alpha T^{2} k^{2}\right]$, where $\alpha$ is the Doppler rate, plus the PN which follows the DVB-S2 compliant model. Code $\mathcal{C}_{3}$ is considered. For the uniformly distributed frequency offset, the range is $\left[-10^{-2}, 10^{-2}\right]$, whereas the number of quantization levels for the proposed receiver is the same as the previous figure. In the figure, the BER at $E_{b} / N_{0}=3.9 \mathrm{~dB}$, for the proposed algorithm and the Tikhonov algorithm enhanced by the RB estimator using a preamble of $P=256$ symbols, is shown as a function of the normalized Doppler rate $\alpha T^{2}$. We may observe that the proposed receiver, for the considered code characterized by very long (therefore, more sensitive) codewords, is able to tolerate values of the Doppler rate $\alpha T^{2} \leq 0.6 \cdot 10^{-7}$. This means that in LEO satellite systems [29], assuming a carrier frequency of at most $60 \mathrm{GHz}$, for values of $1 / T$ larger than $400 \mathrm{kBd}$, the Doppler rate can be tolerated. For lower baud-rate values, simple Doppler rate estimators based on a few symbols can be adopted [30].

To highlight this aspect, in Fig. 6, we show the overall BER curves for the two receivers in Fig. 5 and $\alpha T^{2}=0.6 \cdot 10^{-7}$. We may observe that the receiver based on the RB estimator experiences an error floor, whereas the proposed receiver is almost insensitive to the considered Doppler rate. The reason of this behavior is twofold. For illustration purposes, let us assume absence of PN. In the presence of a Doppler rate, the frequency is not constant, but starting from the value $\nu$, increases linearly with slope $\alpha$. The receiver estimating the frequency on a preamble of $P$ symbols by using the RB algorithm, observes a frequency which starts from the value $\nu$ and reaches the value $\nu+\alpha P T$. As a consequence, it estimates a symbol-rate normalized frequency around $\nu T+\left(\alpha P T^{2} / 2\right)$, which is $\simeq \nu T$ for the considered value of $\alpha$, although during the codeword the frequency increases from the normalized value $\nu T$ to the value $\nu T+\alpha K T^{2}$. Regarding the proposed receiver, as already 


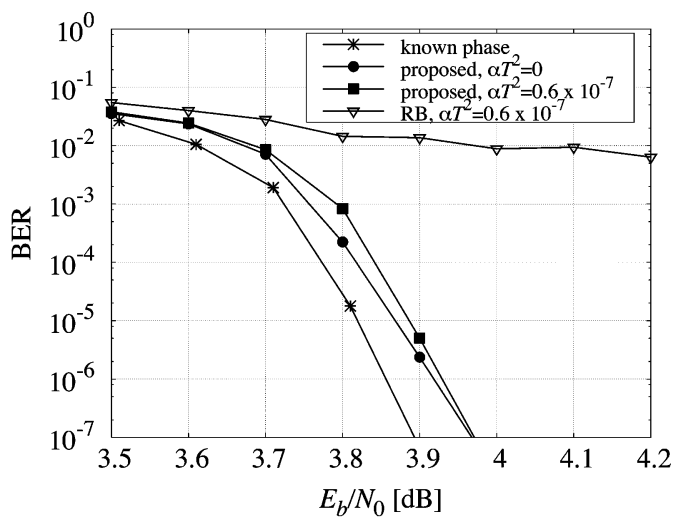

Fig. 6. Performance in the presence of Doppler rate for code $\mathcal{C}_{3}$. The original forward-backward schedule is considered.

mentioned, it does not produce an explicit frequency estimate. Assuming, however, the value of the estimated frequency as the mean value of the estimated frequency pdf, this estimate is based on the whole codeword, since the code symbols become pilots when the iterative decoding process goes on. Hence, the proposed receiver observes the whole evolution of the frequency from the value $\nu$ to the value $\nu T+\alpha K T^{2}$. In addition, although for a given iteration the considered quantization levels remain constant, the corresponding probabilities can change during a codeword. Hence, the algorithm has a (moderate) capability of adaptation.

\section{CONCLUSION}

In this paper, a new algorithm for detection in the presence of random time-varying carrier phase and constant unknown frequency offset has been proposed. The algorithm can be easily embedded in iterative decoding schemes for turbo or LDPC codes, thus performing joint detection and decoding in an iterative fashion.

Despite its very low complexity, the algorithm ensures a very good performance and does not require long training sequences for phase and frequency acquisition and tracking. The advantage, in terms of performance and complexity, with respect to the use of classical solutions based on data-aided or soft-decision-directed separate frequency-estimation algorithms, has also been shown. Finally, the robustness in the presence of a time-varying frequency offset has been also assessed.

\section{APPENDIX}

The following facts are used for the development of the algorithm in Section III. The first two facts may be immediately proved by direct calculation.

Fact 1: Let $t\left(\zeta_{1} ; x\right)$ and $t\left(\zeta_{2} ; x\right)$ be two Tikhonov distributions in $x$, then

$$
t\left(\zeta_{1} ; x\right) t\left(\zeta_{2} ; x\right)=\frac{\mathrm{I}_{0}\left(\left|\zeta_{1}+\zeta_{2}\right|\right)}{2 \pi \mathrm{I}_{0}\left(\left|\zeta_{1}\right|\right) \mathrm{I}_{0}\left(\left|\zeta_{2}\right|\right)} t\left(\zeta_{1}+\zeta_{2} ; x\right)
$$

Fact 2: Let $t(\zeta ; x)$ be a Tikhonov distribution and $\eta$ a real constant, then

$$
t(\zeta ; x+\eta)=t\left(\zeta e^{-j \eta} ; x\right) .
$$

Fact 3: Let $t(\zeta ; y)$ be a Tikhonov distribution in $y$ characterized by a complex constant $\zeta$, and $g\left(y, \sigma^{2} ; x\right)$ a Gaussian distribution with mean value $y$ and variance $\sigma^{2}$, then

$$
\int_{-\pi}^{\pi} t(\zeta ; y) g\left(y, \sigma^{2} ; x\right) d y \simeq t\left(\frac{\zeta}{1+\sigma^{2}|\zeta|} ; x\right) .
$$

Proof: The proof is based on the following approximation which holds for large values of $|\zeta|$ (in practice, $|\zeta|>5$ ):

$$
t(\zeta ; y) \simeq g\left(\arg (\zeta), \frac{1}{|\zeta|} ; y\right)
$$

In fact, for sufficiently large values of $|\zeta|$, the Tikhonov pdf $t(\zeta ; y)$ has its support in a small interval around $\arg (\zeta)$. Hence, by using a second-order Taylor expansion of its argument, we have

$$
\begin{aligned}
t(\zeta ; y) & =\frac{1}{2 \pi \mathrm{I}_{0}(|\zeta|)} e^{\Re\left[\zeta e^{-j y}\right]} \\
& =\frac{1}{2 \pi \mathrm{I}_{0}(|\zeta|)} e^{|\zeta| \cos (y-\arg [\zeta])} \\
& \simeq \frac{1}{2 \pi \mathrm{I}_{0}(|\zeta|)} e^{|\zeta|\left[1-\frac{\left(y-\arg [\zeta \zeta)^{2}\right.}{2}\right]} \\
& =\frac{e^{|\zeta|}}{2 \pi \mathrm{I}_{0}(|\zeta|)} e^{-|\zeta| \frac{\left(y-\arg [\zeta \zeta)^{2}\right.}{2}} \\
& \simeq \sqrt{\frac{|\zeta|}{2 \pi}} e^{-|\zeta| \frac{\left(y-\arg [\zeta \zeta)^{2}\right.}{2}} \\
& =g\left(\arg (\zeta), \frac{1}{|\zeta|} ; y\right)
\end{aligned}
$$

having also used the approximation for large arguments of the modified Bessel function.

Hence, for large arguments of $|\zeta|$, we have

$$
\begin{aligned}
& \int_{-\pi}^{\pi} t(\zeta ; y) g\left(y, \sigma^{2} ; x\right) d y \\
& \quad \stackrel{(a)}{\simeq} \int_{-\infty}^{\infty} t(\zeta ; y) g\left(y, \sigma^{2} ; x\right) d y \\
& \quad \stackrel{(b)}{\simeq} \int_{-\infty}^{\infty} g\left(\arg (\zeta), \frac{1}{|\zeta|} ; y\right) g\left(y, \sigma^{2} ; x\right) d y \\
& \quad \stackrel{(c)}{=} g\left(\arg (\zeta), \sigma^{2}+\frac{1}{|\zeta|} ; x\right) \stackrel{(d)}{\simeq} t\left(\frac{\zeta}{1+\sigma^{2}|\zeta|} ; x\right)
\end{aligned}
$$

where (a) follows from the observation that for large values of $|\zeta|$ or small values of $\sigma$, the product $t(\zeta ; y) g\left(y, \sigma^{2} ; x\right)$ has its support in a small interval, (b) and (d) follow from (26), whereas (c) follows from direct computation.

\section{REFERENCES}

[1] M. Peleg, S. Shamai (Shitz), and S. Gálan, "Iterative decoding for coded noncoherent MPSK communications over phase-noisy AWGN channel," IEE Proc. Commun., vol. 147, pp. 87-95, Apr. 2000.

[2] G. Colavolpe, G. Ferrari, and R. Raheli, "Noncoherent iterative (turbo) detection," IEEE Trans. Commun., vol. 48, no. 9, pp. 1488-1498, Sep. 2000.

[3] A. Anastasopoulos and K. M. Chugg, "Adaptive iterative detection for phase tracking in turbo coded systems," IEEE Trans. Commun., vol. 49, no. 12, pp. 2135-2144, Dec. 2001.

[4] V. Lottici and M. Luise, "Carrier phase recovery for turbo-coded linear modulations," in Proc. IEEE Int. Conf. Commun., New York, NY, Apr. 2002, pp. 1541-1545. 
[5] N. Noels, C. Herzet, A. Dejonghe, V. Lottici, H. Steendam, M. Moeneclaey, M. Luise, and L. Vandendorpe, "Turbo synchronization: An EM algorithm interpretation," in Proc. IEEE Int. Conf. Commun., Anchorage, AK, Jun. 2003, pp. 2933-2937.

[6] V. Lottici and M. Luise, "Embedding carrier-phase recovery into iterative decoding of turbo-coded linear modulations," IEEE Trans. Commun., vol. 52, no. 4, pp. 661-669, Apr. 2004.

[7] A. P. Worthen and W. E. Stark, "Unified design of iterative receivers using factor graphs," IEEE Trans. Inf. Theory, vol. 47, no. 2, pp. 843-849, Feb. 2001

[8] J. Dauwels and H.-A. Loeliger, "Joint decoding and phase estimation: An exercise in factor graphs," in Proc. IEEE Int. Symp. Inf. Theory, Yokohama, Japan, Jun./Jul. 2003, p. 231.

[9] R. Nuriyev and A. Anastasopoulos, "Pilot-symbol-assisted coded transmission over the block-noncoherent AWGN channel," IEEE Trans. Commun., vol. 51, no. 6, pp. 953-963, Jun. 2003.

[10] J. Dauwels and H.-A. Loeliger, "Phase estimation by message passing," in Proc. IEEE Int. Conf. Commun., Paris, France, Jun. 2004, pp. $523-527$.

[11] G. Colavolpe, "LDPC codes over channels with memory"' in Proc. IEEE Int. Conf. Commun., Paris, France, Jun. 2004, pp. 757-761.

[12] — "On LDPC codes over channels with memory," IEEE Trans. Wireless Commun., to be published.

[13] G. Colavolpe, A. Barbieri, and G. Caire, "Algorithms for iterative decoding in the presence of strong phase noise," IEEE J. Sel. Areas Commun., vol. 23, no. 9, pp. 1748-1757, Sep. 2005.

[14] S. L. Howard and C. Schlegel, "Differential turbo coded modulation with APP channel estimation," IEEE Trans. Commun., to be published.

[15] F. R. Kschischang, B. J. Frey, and H.-A. Loeliger, "Factor graphs and the sum-product algorithm," IEEE Trans. Inf. Theory, vol. 47, no. 2, pp. 498-519, Feb. 2001.

[16] J. Dauwels, H. Wymeersch, H.-A. Loeliger, and M. Moeneclaey, "Phase estimation and phase ambiguity resolution by message passing," in Proc. 11th Int. Conf. Telecommun. Netw., Fortaleza, Brasil, Aug. 2004, pp. 150-155.

[17] U. Mengali and A. N. D. Andrea, Synchronization Techniques for Digital Receivers (Applications of Communications Theory). New York: Plenum, 1997.

[18] M. Morelli and U. Mengali, "Feedforward frequency estimation for PSK: A tutorial review," Eur. Trans. Telecommun., vol. 9, pp. 103-116, Mar./Apr. 1998.

[19] D. C. Rife and R. R. Boorstyn, "Single tone parameter estimation from discrete-time observations," IEEE Trans. Inf. Theory, vol. IT-20, no. 7, pp. 591-598, Sep. 1974

[20] M. Luise and R. Reggiannini, "Carrier frequency recovery in all-digital modems for burst-mode transmissions," IEEE Trans. Commun., vol. 43, no. 3, pp. 1169-1178, Mar. 1995.

[21] U. Mengali and M. Morelli, "Data-aided frequency estimation for burst digital transmission," IEEE Trans. Commun., vol. 45, no. 1, pp. 23-25, Jan. 1997.

[22] N. Noels, H. Steendam, and M. Moeneclaey, "Pilot-symbol assisted iterative carrier synchronization for burst transmission," in Proc. IEEE Int. Conf. Commun., Paris, France, Jun. 2004, pp. 509-513.

[23] A. Ginesi, D. Fittipaldi, A. Bigi, and R. De Gaudenzi, "Pilot-aided carrier synchronization techniques for broadband satellite transmissions," ESA-ESTEC Tech. Rep., Sep. 2003.

[24] D. J. C. MacKay, Regular LDPC online database [Online]. Available: http://www.inference.phy.cam.ac.uk/mackay/

[25] Digital Video Broadcasting (DVB): Second Generation Framing Structure, Channel Coding and Modulation Systems for Braoadcasting, Interactive Services, News Gathering and Other Broadband Satellite Applications, ETSI DVBS2 74r13, 2003.

[26] J. A. Gansman, J. V. Krogmeier, and M. P. Fitz, "Single frequency estimation with non-uniform sampling," in Proc. Asilomar Conf. Signals, Syst., Comput., Nov. 1997, pp. 399-403.

[27] S. M. Kay, "A fast and accurate single frequency estimator," IEEE Trans. Acoust., Speech, Signal Process., vol. 37, no. 6, pp. 1987-1990, Dec. 1989.

[28] A. Barbieri, D. Bolletta, and G. Colavolpe, "On the Cramer-Rao bound for carrier frequency estimation in the presence of phase noise," in Proc. IEEE Global Telecommun. Conf., St. Louis, MO, 2005, pp. $1441-1445$.

[29] I. Ali, N. Al-Dhahir, and J. E. Hershey, "Doppler characterization for LEO satellites," IEEE Trans. Commun., vol. 46, no. 3, pp. 309-313, Mar. 1998.

[30] M. Morelli, "Doppler-rate estimation for burst digital transmission," IEEE Trans. Commun., vol. 50, no. 5, pp. 707-710, May 2002.

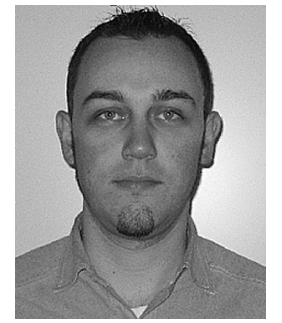

Alan Barbieri (S'05) was born in Parma, Italy, in 1979. He received the Dr. Ing. degree in telecommunications engineering (cum laude) in 2003 from the University of Parma, Parma, Italy, where he is currently working towards the Ph.D. degree in the $\mathrm{Di}$ partimento di Ingegneria dell' Informazione.

His main research interests include digital transmission theory and information theory, with particular emphasis on channel coding, iterative joint detection and decoding algorithms, estimation of unknown parameters, and algorithms for synchronization. He participates in several research projects funded by the European Space Agency (ESA-ESTEC) and important telecommunications companies.

Mr. Barbieri was the recipient of the Premio Conti for the year 2003, as the best graduate in Information Engineering at the University of Parma in the academic year 2003.

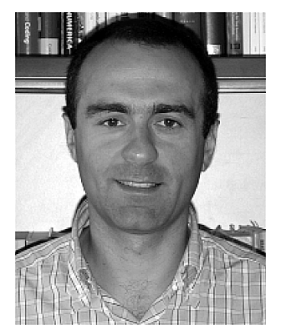

Giulio Colavolpe was born in Cosenza, Italy, in 1969. He received the Dr. Ing. degree in telecommunications engineering (cum laude) from the University of Pisa, Pisa, Italy, in 1994, and the $\mathrm{Ph} . \mathrm{D}$. degree in information technology from the University of Parma, Parma, Italy, in 1998.

Since 1997, he has been with the University of Parma, Italy, where he is now an Associate Professor of Telecommunications. In 2000, he was a Visiting Scientist at the Institut Eurécom, Valbonne, France. His main research interests include digital transmission theory, channel coding, and signal processing. His research activity has led to several scientific publications in leading international journals and conference proceedings and a few industrial patents. He is also coauthor of the book Detection Algorithms for Wireless Communications (New York: Wiley, 2004). $\mathrm{He}$ is also the principal investigator of several research projects funded by the European Space Agency (ESA-ESTEC) and important telecommunications companies.

In 2005, Dr. Colavolpe received the Best Paper Award for the paper "VLSI Design of a Fully Parallel Decoder for LDPC-like Codes," at the 13th International Conference on Software, Telecommunications and Computer Networks (SoftCOM 2005).

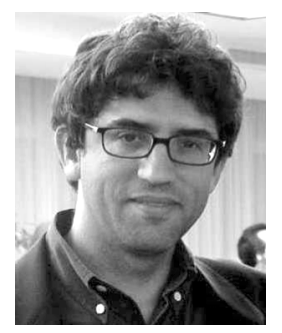

Giuseppe Caire (S'92-M'94-SM'03-F'05) was born in Torino, Italy, in 1965. He received the B.Sc. degree in electrical engineering from the Politecnico di Torino, Torino, Italy, in 1990, the M.Sc. degree in electrical engineering from Princeton University, Princeton, NJ, in 1992, and the Ph.D. degree from the Politecnico di Torino in 1994.

$\mathrm{He}$ was with the European Space Agency (ESTEC), Noordwijk, The Netherlands, from May 1994 to February 1995. He was visiting Princeton University in the summer of 1997 and Sydney University, Sydney, Australia, in the summer of 2000. He was an Assistant Professor in Telecommunications with the Politecnico di Torino, an Associate Professor with the University of Parma, Parma, Italy, a Professor with the Department of Mobile Communications, Eurecom Institute, Sophia Antipolis, France, and he is currently a Professor with the Electrical Engineering Department, University of Southern California, Los Angeles, CA. His current interests are in the field of communications theory, information theory, and coding theory, with particular focus on wireless applications.

Dr. Caire was a recipient of the AEI G. Someda Scholarship in 1991, was a recipient of the COTRAO Scholarship in 1996, and of a CNR Scholarship in 1997. He served as an Associate Editor for the IEEE TRANSACTIONS ON COMMUNICATIONS in 1998-2001, and as an Associate Editor for the IEEE TRANSACTIONS ON INFORMATION THEORY in 2001-2003. He received the Jack Neubauer Best System Paper Award from the IEEE Vehicular Technology Society in 2003, and the Joint IT/Comsoc Best Paper Award in 2004. Since November 2004, he has been a member of the Board of Governors of the IEEE Information Theory Society. 